(C) 2010 IEEE. Personal use of this material is permitted. Permission from IEEE must be obtained for all other uses, in any current or future media, including reprinting/republishing this material for advertising or promotional purposes, creating new collective works, for resale or redistribution to servers or lists, or reuse of any copyrighted component of this work in other works. 


\title{
Arm-Hand Movement: Imitation of Human Natural Gestures with Tenodesis Effect
}

\author{
Kien-Cuong Nguyen and Véronique Perdereau
}

\begin{abstract}
For an anthropomorphic arm-hand robot, grasping and in-hand manipulating an object can be realized with numerous approach trajectories and grasping configurations. The redundancy at this level of the tasks is due to a large number of degrees-of-freedom (DOFs) of the arm-hand system. This redundancy constitutes a big challenge to the planning and control tasks of the robot. For this kind of tasks, human has his own choices privileging certain configurations over the others. These choices come from a long learning process which implicitly takes into account the mechanical constraints of the system. In this work, we concentrate our effort on deciphering certain mechanical constraints, "tenodesis" phenomenon in particular, in order to solve the redundancy and imitate the human natural gestures in the tasks of grasping or in-hand manipulation.
\end{abstract}

\section{INTRODUCTION}

Human-object interaction appears to be a very complex task, particularly due to the high redundancy of the armhand system and the multiplicity of ways to approach an object. With such redundancy and multiplicity, humans have many choices to approach, grasp and hold an object in the hand even when the position of the object and the grasp configuration are specified. However, humans tend to, apparently, choose only certain configurations for a specific grasp. By closely observing how humans act, two factors (mechanisms) that could significantly impact the choices of humans in such situation are identified:

- Experience acquired over time through learning processes: experience helps human choose cylindrical grasp for a bottle, tripod grasp for table-tennis ball...

- Mechanical constraints of the arm-hand system: when doing the tasks of grasping and in-hand manipulation, human tends to automatically optimize different mechanical criteria such as stability, manipulability, comfort...

In this paper, we concentrate our efforts on deciphering the mechanical constraints of the human arm-hand system for the tasks of grasping and in-hand manipulation, justifying from the mechanical point of view the human choices while performing such tasks. As a human hand is an exemplary gripper, a dexterous manipulator and that our Shadow armhand robot is morphologically very closed to it, the knowledge of these constraints will be very helpful to the robot

UPMC Univ Paris 06, UMR7222, ISIR, F-75005, Paris, France, kien-cuong.nguyen@upmc.fr

The research leading to these results has been supported by the HANDLE project, which has received funding from the European Communitys Seventh Framework Programme (FP7/2007-2013) under grant agreement ICT 231640 . planning and control, and in particular, to the resolution of the redundancy problem.

The mechanical constraint that is particularly studied in this paper is the tendon constraint of the hand, or the "tenodesis effect" in the language of bio-mechanical researchers. This constraint is simply stated in biomechanics as induced movements of finger joints when the wrist joint moves (in relaxing mode). This article further clarifies the tenodesis phenomenon by:

- Constructing a simplified mechanical model of the hand,

- Resolving the model to predict joint-angle variation by using optimization method with an introduction to "muscle comfort" criterion,

- Validating the model by using data recorded from human actions.

The muscle comfort criterion developed above is then integrated to a framework of redundancy resolution for the grasping and in-hand manipulation tasks with an anthropomorphic arm-hand robot. The obtained results are compared with human movements to check if the "natural characteristics" of the gestures are reproduced.

The article is structured as follows: we present in detail the description of the tenodesis phenomenon and the studies with challenges up to date on this phenomenon in section II. It is followed by our assumption on human hand natural gestures in which tenodesis effect plays an important role. Section III begins with a simplified model of the hand expressed in the form of hypothesis. This simplified model constitutes the base for our later analysis. The "muscle comfort" criterion mentioned in previous section is then quantitatively developed. The theoretical and experimental validation of this criterion is presented at the end of section IV. Several direct applications of the "muscle comfort" criterion are given in section V. Conclusion and future works are presented in section VI.

\section{TENODESIS EFFECT - ASSUMPTION ON HUMAN HAND NATURAL GESTURES}

Tenodesis effect is a particularly interesting phenomenon and has important impacts on the natural gestures of the hand. This section begins with the description of the phenomenon and the studies available in the literature up to this date. It is followed by our assumption on human hand natural gestures and the role of tenodesis effect in such gestures.

\section{A. Tenodesis Effect}

The tenodesis effect is described by bio-mechanical researchers as the change in the finger joints induced by 
the wrist joint movement (Fig. 1). This phenomenon is due to the fact that extensor digitorum communis (EDC), interossei (IO), flexor digitorum profundus (FDP) and flexor digitorum sublimis (FDS) tendons go through the wrist joint [1]. These passages constitute additional tendon constraints in the hand and produce correlations between wrist joint and finger joints. These correlations sometimes are named as wrist synergistic movements.

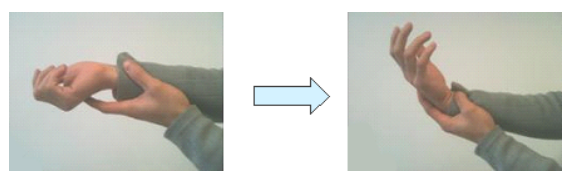

Fig. 1. Tenodesis Effect.

The correlations described above exist in both passive movements (the hand is totally relaxed and the wrist joint angle changes passively as show in the Fig. 1) and active movements (when fingertips try to reach some predefined points in space, the joint angles do not take arbitrary values but only some with certain correlations between them). However, the quantitative study of those correlations in active movements appears to be very difficult to conduct. For the correlations in passive movements, there are several quantitative studies that have already been done in the domain of biomechanics. In $\mathrm{Su}$, et al. [2], the authors make an approximation of finger joint movements by a second degree polynomial of wrist joint.

That study proves that there is a correlation between the finger joints and the wrist joint in passive movements and presents a first quantitative estimation of such correlations. However there are a number of limits in the study:

- Imprecise estimation: as shown in [2], the estimation is not very precise and is not usable to predict the movement of a particular arm-hand system.

- Simple statistical method only: the study simply uses quadratic regression for two variables (finger joint angle and wrist joint angle) whereas hand tendon system is much more complicated than that. This is one of the reasons why the estimation is not very precise.

- Limited to passive movements: the presented method cannot be used to study the correlations in active movements.

- Result formulation: the obtained results are formulated in the form of linear or quadratic relation between a certain finger joint angle and the wrist joint angle. This type of formulation cannot directly be applied to the active motion because it involves multi-joint angles.

These limits lead us to take another approach that will be presented in the following subsections.

\section{B. Assumption on human hand natural gestures}

By closely observing the hand natural gestures, we find out that the hand configuration always stays "closest" in some way to the totally relaxed state. More precisely, when performing a task such as fixing the fingertips at a specific position, human hand tends to choose a certain configuration that we call it a "natural" one. By maintaining this task and deviating other parts of the hand (wrist, palm, etc.) from the "natural" configuration, we begin to feel uncomfortable. The more we deviate the hand from this "natural" configuration (but always maintain the mentioned task: fixing the fingertips at a specific position), the more uncomfortable we feel. This discomfort may come from different sources: unfamiliarity with the new configuration; feeling of unstable, less control; over-contraction of veins, nerves, etc. However, in the case of human hand, the main reason could be the "impact" of the hand configuration on the functioning of the muscle system or the degree of "comfort" of this one. And this constitutes our assumption on the human hand natural gestures.

Assumption 2.1: In case of redundancy for a specific task, the natural configuration of the hand is the one that maximize the "comfort" of the muscle system.

The quantitative analysis of the muscle comfort is given in section III. The tenodesis effect and the hand passive movement constitute an excellent base to validate this concept.

\section{MUSCLE COMFORT CRITERION}

Before quantitatively developing the muscle comfort criterion and validating it, we present here a simplified model of the hand on which we establish our analysis.

\section{A. Simplified Model of the Hand}

As many other limbs, human hands are composed of skeletons (connected by ligaments to form the joints), muscles, tendons, vein, skin, etc. Each element has its own way to impact the kinematics of the hand but the skeleton structure, muscles and the tendons appear to be the most important.

In order to quantitatively analyze, predict, estimate the dynamic and kinematic quantities of the arm-hand system, biomechanical models should be elaborated. For this part of body, there are numerous models created until this date: for muscles models, we have the ones of Hill [3] and Zajac [4]; for joints structure, we have the model of Hollister [5]; for tendon excursion, we have the one presented in [6].

In the goal of better analyzing the tenodesis effect and for computational reasons, we simplify the above biomechanical models and focus only on the aspects concerning this phenomenon. We formulate our model in the form of hypothesis for the easiness of referencing.

The first hypothesis presents the passive force - muscle deformation relation adopted in this article. This hypothesis is a simplified version of the formula presented in [3] where only the passive force is taken into consideration.

Hypothesis 3.1: The muscle passive force $F^{P M}$ of a muscle $m$ with length $l$ and optimal length $l_{0}$ is given by $F^{P M}=F_{\max } f_{p}(\varepsilon)$, where $\varepsilon=\left(l-l_{0}\right) / l_{0}$ is the muscle deformation and $f_{p}(\varepsilon)=c_{1} e^{c_{2} \varepsilon}$ is the passive force-muscle deformation function.

The passive force function $f_{p}(\varepsilon)$ in this hypothesis is an approximation of the one given in [3]. This function is simpler to calculate and it is closer to zero when $\varepsilon<0$. The two constants $c_{1}$ and $c_{2}$ are chosen to be 0.012 and 7.5 respectively so that $f_{p}$ is the closest to the one in [3] according to the $L_{2}$ norm. 
The second hypothesis concerns the tendon excursion. We suppose that the tendons run around the joints of circular forms (pulleys) and are always attached to them as shown in Fig. 2 where the extension tendon (red) runs around the pulley of radius $R_{e 1}$ and the flexion tendon (green) runs around the pulley of radius $R_{f 1}$.

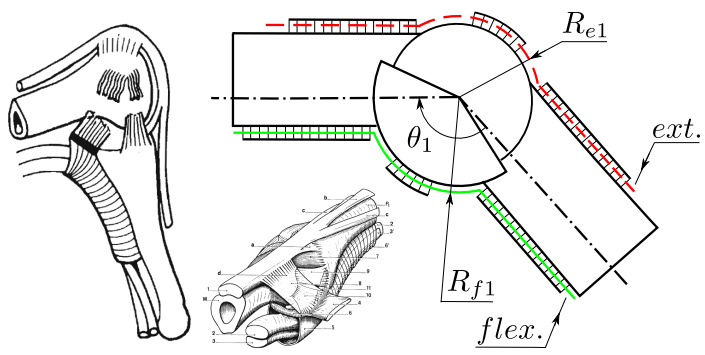

Fig. 2. Tendon excursion model (extension tendon: red dashed line; and flexion tendon: green continuous line).

Hypothesis 3.2: The variation of excursion $\Delta \varepsilon$ of the tendon $t$ through the joint $j$ is proportional to the joint angle variation $\Delta \theta$ and the proportional coefficient is $\pm R_{t, j}$ (pulley radius to which the tendon attaches). More precisely, $\Delta \varepsilon_{t j}= \pm R_{t j} \Delta \theta_{j}$.

In the case of flexion and extension tendons presented in the Fig. 2, we have: $\Delta \varepsilon_{e 1}=-R_{e 1} \Delta \theta_{1}$ and $\Delta \varepsilon_{f 1}=$ $+R_{f 1} \Delta \theta_{1}$.

As the weight of finger-bones is small compared to muscle efforts, we neglect the impact of gravity in this model.

Hypothesis 3.3: The impact of gravity is negligible in this model.

The last hypothesis concerns the friction in joints and tendon passage.

Hypothesis 3.4: The friction in joints and tendon passage is supposed to be negligible.

\section{B. Quantitative Definition of Muscle Comfort}

After the Hill model [3], a muscle $m$ at relaxed state (no activation) changes its tension $F_{m}$ in function of its length $l_{m}$. We can then associate a potential energy $\mathcal{E}_{P}$ to the muscle in the following form:

$$
\mathcal{E}_{P}\left(l_{m}\right)=-\int_{0}^{l_{m}} \overrightarrow{F_{m}(x)} \overrightarrow{d x}=\int_{0}^{l_{m}} F_{m}(x) d x .
$$

For a real muscle, the tension is zero when its length (distance between its two tips) is smaller than its optimal length $l_{0}$. This tension increases when its length $l_{m}$ increases. As the more this tension increases, the more we feel uncomfortable, we call then the "comfort" of the muscle the following quantity

$$
\mathcal{C}_{m}\left(l_{m}\right)=A-\mathcal{E}_{P}\left(l_{m}\right)=A-\int_{0}^{l_{m}} F_{m}(x) d x,
$$

where $A$ is a constant. It is evident from this expression that the "comfort" quantity decreases when the muscle is stretched to a length greater then $l_{0}$.

For a set $\mathrm{M}$ of muscles:

$$
\mathcal{C}_{\mathrm{M}}(l)=\sum_{m \in \mathrm{M}} \mathcal{C}_{m}\left(l_{m}\right)
$$

is the muscle comfort of this set (we denote here $l=$ $\left.\left(l_{m}\right)_{m \in \mathrm{M}}\right)$.
The muscle comfort is the key element in our redundancy resolution algorithm which helps retrieving the natural configuration of human gestures. The following conjecture describes its role in human hand movements.

Conjuncture 3.1: In relaxing mode, the hand takes a configuration that maximizes its muscle comfort:

$$
\left(\theta_{i}\right)_{\text {relaxed }}=\underset{\left(\theta_{i}\right)}{\operatorname{argmax}} \mathcal{C}_{\mathrm{H}}=\underset{\left(\theta_{i}\right)}{\operatorname{argmax}} \sum_{m \in \mathrm{H}} \mathcal{C}_{m},
$$

where $\mathrm{H}$ is the set of all muscles in the hand and forearm.

The conclusion of this conjecture gets the inspiration from the system of two springs connected in series (Fig. 3) even though the human hand structure is highly complicated, it is essentially composed of antagonistic muscles.

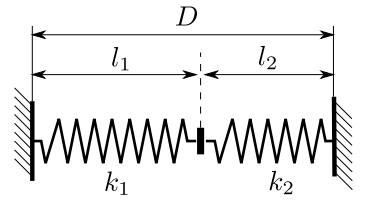

Fig. 3. System of two springs connected in series.

Indeed, in the case of two springs connected in series (Fig. 3 ), the system is in the balance state when their "comfort" is maximized:

$$
\left(l_{1_{\text {balanced }}}, l_{2_{\text {balanced }}}\right)=\underset{\left\{l_{1}, l_{2}: l_{1}+l_{2}=D\right\}}{\operatorname{argmax}}\left(\mathcal{C}_{s_{1}}\left(l_{1}\right)+\mathcal{C}_{s_{2}}\left(l_{2}\right)\right) .
$$

as their "comfort" is the opposite of their potential energy (after 2) and as these two springs are balanced when their potential energy is minimized.

It is worth noting, however, that the passive behavior of the muscles is not the same as the one of springs (hypothesis 3.1) and that the hand musculo-tendon structure is much more complicated then the system of two springs. The following subsections examine in detail this conjuncture and validate it both theoretically and experimentally.

\section{VALIDATION}

\section{A. Theoretical Validation}

In order to theoretically validate the conjuncture 3.1 on the principle of muscle comfort maximization, we show that the results produced by (4) are exactly the same as the ones produced by the dynamic equations in the case of quasi-static passive movement of the hand (the passive form in which the tenodesis appears presented in subsection II-A).

1) Quasi-static equations: In the quasi-static movement, the velocity and acceleration of the elements are negligible. The movement is considered as a sequence of stable states. As the forearm-hand system is constituted of solid bones linked by solid joints, it is sufficient to consider the nullity of the torque applied on each bone relative to a joint axis.

Let consider the distal-phalanx $\mathcal{P}_{3}$ of the first-finger in the first place. There are two tendons (EDC or $e$ and FDP or $p$ ) attached to this phalanx and controlling the motion of it (Fig. 4). As gravity and friction are negligible, there are only three significant forces applied on this phalanx: $e$ tendon force $\overrightarrow{F_{e 3}}, p$ tendon force $\overrightarrow{F_{p 3}}$ and inter-phalanx force $\overrightarrow{F_{23}}$. The stability of the distal-phalanx implies that the sum of the applied forces and torques are zero: 


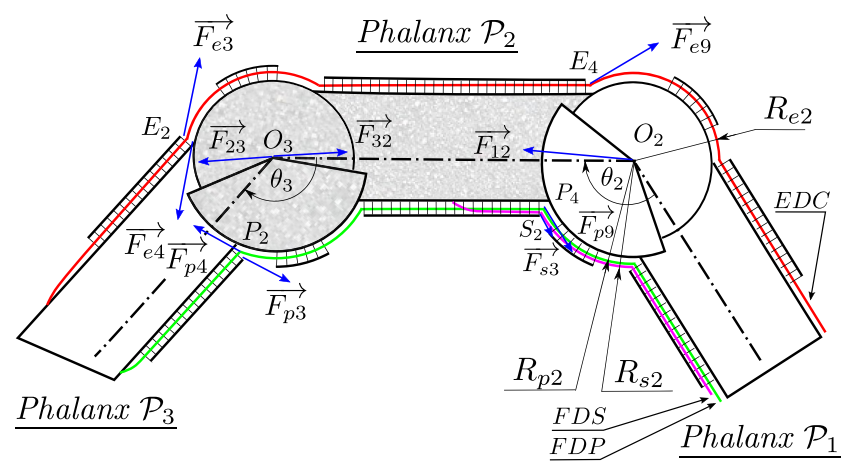

Fig. 4. Schema of forces applied on the distal $\mathcal{P}_{3}$ and inter phalanx $\mathcal{P}_{2}$ (red: $E D C$ tendon, green: $F D P$ tendon and pink: $F D S$ tendon).

$$
\begin{aligned}
& \overrightarrow{0}=\overrightarrow{F_{e 3}}+\overrightarrow{F_{p 3}}+\overrightarrow{F_{23}} \\
& \overrightarrow{0}=\overrightarrow{O_{3} E_{2}} \times \overrightarrow{F_{e 3}}+\overrightarrow{O_{3} P_{2}} \times \overrightarrow{F_{p 3}} .
\end{aligned}
$$

where $\times$ is the cross product of two vectors. As all the forces are in the same plane, $(5 \mathrm{~b})$ can be rewritten as follows:

$$
0=R_{p 3} F_{p}-R_{e 3} F_{e 3} .
$$

For the inter-phalanx $\mathcal{P}_{2}$, there are two tendons ( $E D C$ or $e$ and $F D P$ or $p$ ) going through it and an other ( $F D S$ or $s$ ) inserting to it (Fig. 4). By the same argument as in the previous case, there are only 7 significant forces applied on this phalanx: $e$ tendon forces $\left(\overrightarrow{F_{e 4}}\right.$ and $\left.\overrightarrow{F_{e 9}}\right), p$ tendon forces $\left(\overrightarrow{F_{p 4}}\right.$ and $\left.\overrightarrow{F_{p 9}}\right), s$ tendon force $\overrightarrow{F_{s 2}}$, distal and proximalphalanx forces $\left(\overrightarrow{F_{32}}\right.$ and $\left.\overrightarrow{F_{12}}\right)$. By taking into account $(5)$ and the fact that $\overrightarrow{F_{e 4}}=-\overrightarrow{F_{e 3}}, \overrightarrow{F_{p 4}}=-\overrightarrow{F_{p 3}}, \overrightarrow{F_{32}}=-\overrightarrow{F_{23}}$, the stability of the inter-phalanx implies that:

$$
\begin{aligned}
\overrightarrow{0} & =\overrightarrow{F_{e 9}}+\overrightarrow{F_{p 9}}+\overrightarrow{F_{s 3}}+\overrightarrow{F_{12}} \\
\overrightarrow{0} & =\overrightarrow{O_{2} E_{4}} \times \overrightarrow{F_{e 9}}+\overrightarrow{O_{2} P_{4}} \times \overrightarrow{F_{p 9}}+\overrightarrow{O_{2} S_{2}} \times \overrightarrow{F_{s 3}}
\end{aligned}
$$

Equation (7b) can be rewritten as follows:

$$
0=R_{p 2} F_{p}+R_{s 2} F_{s}-R_{e 2} F_{e 9}
$$

The same procedure can be applied to the proximal-phalanx and other bones to obtain other quasi-static equations.

2) Equivalence of the two approaches: We demonstrate in this section that the joint-angles resulted from the conjuncture 3.1, equation (4), also satisfy the quasi-static equations established above. Indeed, suppose that $\left(\theta_{i}\right)_{r}$ is the joint configuration that maximizes the muscle comfort (4), let consider the joint $O_{3}$, the distal phalanx and the tendon forces applied to it (Fig. 4). As $\left(\theta_{i}\right)_{r}$ is the optimal solution, the following equation hold true:

$$
0=\frac{\partial \mathcal{C}_{\mathrm{H}}}{\partial \theta_{3}}\left(\theta_{i}\right)_{r}=\frac{\partial \mathcal{C}_{e}}{\partial \theta_{3}}\left(\theta_{i}\right)_{r}+\frac{\partial \mathcal{C}_{p}}{\partial \theta_{3}}\left(\theta_{i}\right)_{r} .
$$

After the definition of the muscle comfort, and as $\frac{\mathrm{d} l_{e}}{\mathrm{~d} \theta_{3}}=$ $-R_{e 3}$ after hypothesis 3.2 , we have $\frac{\partial \mathcal{C}_{e}}{\partial \theta_{3}}=\frac{\mathrm{d} \mathcal{C}_{e}}{\mathrm{~d} l_{e}} \frac{\mathrm{d} l_{e}}{\mathrm{~d} \theta_{3}}=$ $-F_{e} R_{e 3}$. By the same argument, we obtain: $\frac{\partial \mathcal{C}_{p}}{\partial \theta_{3}}=F_{p} R_{p 3}$. These three equations give us the same equality as in (6). And by the same procedure, we obtain the same equality as in (8) for the inter-phalanx and those for other bones. In other words, $\left(\theta_{i}\right)_{r}$ is the solution of the quasi-static equations.

Conversely, suppose that $\left(\theta_{i}\right)_{d}$ is a solution of the quasistatic equations of the hand. By the same calculations shown above, we deduce that $\frac{\partial \mathcal{C}_{\mathrm{H}}}{\partial \theta_{3}}\left(\theta_{i}\right)_{d}=0, \frac{\partial \mathcal{C}_{\mathrm{H}}}{\partial \theta_{2}}\left(\theta_{i}\right)_{d}=0 \ldots$ As $\mathcal{C}_{\mathrm{H}}$ is a concave function of $\left(\theta_{i}\right),\left(\theta_{i}\right)_{d}$ maximizes then the muscle comfort of the hand $\mathcal{C}_{\mathrm{H}}$. The equivalence of the two approaches is then demonstrated.

\section{B. Experimental Validation}

For the experimental validation of the conjuncture 3.1, we recorded human hand movements and compared the recorded data to the predicted one. The details of the validation process along with the obtained results are presented in the following subsections.

1) Recording Platform: The recording platform that we used in this validation process consists of a Cyberglove II (22 joint angle sensors), a motion tracking sensor (Polhemus Liberty) and a mono camera as shown in Fig. 5(a). This platform locates at FCTUC (Faculty of Science and Technology, University of Coimbra, Portugal). The recorded data from the different devices are stored in XML files and synchronized by using a timestamp parameter.

2) Method: The main idea of the validation process is to compare the predicted joint angle sequence with the recorded one from the human hand. In this process, the human hand is in a totally relaxed state and the wrist joint is moved passively as shown in Fig. 5(b). The recorded data in this step is then divided into two independent parts: one for parameter identification and the other for model validation.

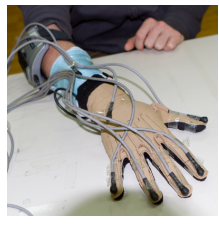

(a)

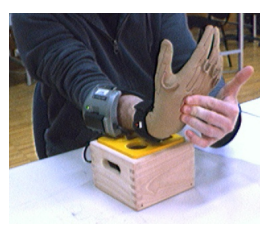

(b)
Fig. 5. Recording platform with Cyberglove and Polhemus Liberty sensors (a) and recording of the hand passive movement (b)

For the first part, we use a genetic algorithm to identify different parameters (muscle optimal lengths $l_{0}$, muscle maximal forces $F_{\max }$, virtual pulley radius $R_{m, i}$ ) that are presented in section III-A. The fitness function used in this algorithm is calculated as follows:

$\operatorname{fitness}\left(R_{m, i}, F_{\max , m}, l_{0, m}\right)=\left\|\mathbf{S}_{\theta, \text { recorded }}-\mathbf{S}_{\theta, \text { predicted }}\right\|$, where $\mathbf{S}_{\theta}$ is a sequence of finger joint angles and $\|\|=$. $\|.\|_{\infty}$, a vectorial norm that is defined as $\left\|\left(x_{1}, \ldots, x_{n}\right)\right\|_{\infty}=$ $\max \left\{\left|x_{1}\right|, \ldots,\left|x_{n}\right|\right\}$. The initial population in this algorithm is set to 20000 and the to-be-determined parameters, muscle optimal lengths and maximal muscle forces in particular, are taken around the values given by Weijs [7], and Garner [8]. The table I gives the obtained results for the first finger.

3) Results: Once the parameters are identified, we use the model to predict the hand passive movements and compare them to the data recorded in the second part. The joint angles of the fingers (first-finger, middle-finger, ring-finger, 
TABLE I

IDENTIFIED PARAMETERS OF FIRST-FINGER

\begin{tabular}{|lc|lc|ll|ll|}
\hline$F_{\max }$ & $(N)$ & $l_{0}$ & $(\mathrm{~cm})$ & $R m, i$ & $(\mathrm{~cm})$ & $R m, i$ & $(\mathrm{~cm})$ \\
\hline$F_{\max , e}$ & 709 & $l_{0, e}$ & 5.7 & $R_{e, 1}$ & 1.50 & $R_{p, 1}$ & 0.90 \\
$F_{\max , p}$ & 677 & $l_{0, p}$ & 6.6 & $R_{e, 2}$ & 0.57 & $R_{p, 2}$ & 0.56 \\
$F_{\max , s}$ & 701 & $l_{0, s}$ & 6.6 & $R_{e, 3}$ & 1.29 & $R_{p, 3}$ & 1.30 \\
$F_{\max , l}$ & 33 & $l_{0, l}$ & 7.3 & $R_{e, 4}$ & 0.76 & $R_{p, 4}$ & 1.99 \\
\hline
\end{tabular}

and little-finger) are calculated in function of the wrist-joint angle. As shown in Fig. 6, the predicted first-finger joint angles are very close to the recorded ones. The established model is then validated for the passive movement of the hand.

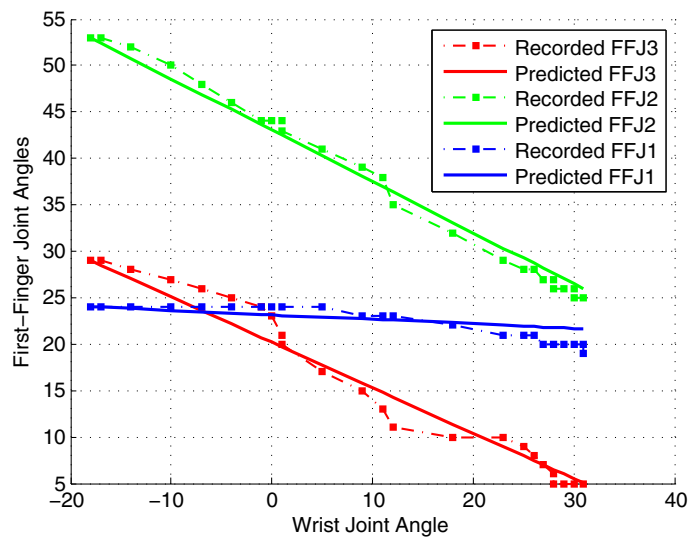

Fig. 6. Comparison between recorded and predicted data for wrist - first finger passive movements: red- $\theta_{1}$, green- $\theta_{2}$, blue- $\theta_{3}$.

\section{APPLICATION}

In contexts like grasping and in-hand manipulation, sometimes the object possesses certain symmetries. Consequently, there are a number of palm position choices for a specific finger configuration in order to realize a grasp. The "can grasping" situation shown in Fig. 7 is a typical example. For an anthropomorphic robot hand, this situation of redundancy could make the robot confused, not knowing which palm position it should take.

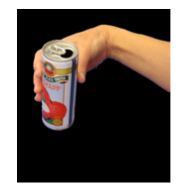

(a)

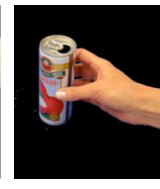

(b)

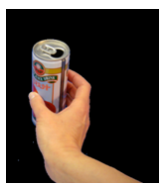

(c)
Fig. 7. Human can grasping

We show in this section the effectiveness of the muscle comfort criterion in solving the redundancy in such situations: the easiness of the resolution and the "natural character" of the gestures. For this objective, we firstly present the simulation environment and the simulated robot with which we work. The redundancy resolution framework and the simulated results come out just after.

\section{A. Simulation environment and simulated robot}

The simulation environment that we use for this work is the Marilou Robotics Studio of the Anykode company [9]. The simulated robot is composed of a hand (same kinematic as the 24 DOFs C6 Shadow Hand [10]) and an arm (same morphology as the 4 DOFs Shadow arm but one fourth in dimension) as shown in Fig. 8.

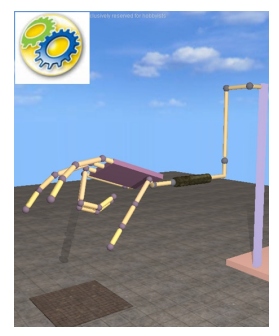

(a)

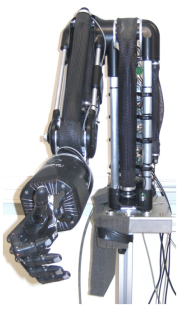

(b)
Fig. 8. Marilou simulation environment with simulated robot (a) and real Shadow arm-hand robot (b)

\section{B. Redundancy Resolution Framework}

We adopt in this paper the redundancy resolution framework presented by Seraji H. [11]. The main idea of this method is to give additional tasks to the redundant system until it is not redundant anymore. More precisely, the final task of a $n$-rigid joint robot will be written in the following form:

$$
X=\left[\begin{array}{l}
Y \\
Z
\end{array}\right]=\left[\begin{array}{l}
f(\theta) \\
g(\theta)
\end{array}\right],
$$

where $Y=f(\theta)$ is the principal task ( $Y$ is a $m \times 1$ vector with $m<n$ ) and $Z=g(\theta)$ represents the additional tasks $(Z$ is a $(n-m) \times 1$ vector). The additional tasks can be in different forms: kinematic optimization, posture control or inequality constraints... In this redundancy resolution framework, the muscle comfort criterion lies in the optimization category which is sometimes called "null space optimization" and often applied with a default posture where the optimization tries to keep the robot configuration as close as possible to this one.

The advantage of the muscle comfort criterion over other types of "default postures" is, as shown in the following section, that the resolved configurations are very close to the human natural gestures (which are apparently unknown analytically).

\section{Simulated results}

By applying the redundancy resolution framework presented above with the muscle comfort optimization as secondary task, we succeeded in solving the redundancy and choosing the "best" palm position in different situations of grasp, among which two (tripod and cylindrical) of them are presented in this subsection.

1) Tripod grasp of a bottle capsule: Tripod is a grasp in which only three fingers are involved, usually thumb, index and middle finger. This grasp is often used for small objects. In the situation of tripod-grasping a bottle capsule with downward approaching direction (Fig. 9), the symmetry of the capsule makes the degree-of-redundancy of the arm increase by one (the palm can take whatever orientation around the symmetry axis when realizing the grasp).

2) Cylindrical grasp of a can: The same redundancy reproduces in the can - cylindrical grasping situation with horizontal approaching direction. The symmetry of the can makes the degree-of-redundancy of the arm increase by one (Fig. 10). 
The redundancy in these two situations are solved by taking the redundancy resolution framework presented in subsection V-B with reaching grasp-center position and graspaxis direction (5 DOFs) as primary task, and maximizing the muscle comfort of the hand (1 DOFs) as secondary task.

In the tripod grasp of bottle capsule, as the joint angles of the arm are limited, the palm direction can only vary from $30^{\circ}$ to $60^{\circ}$ and the corresponding muscle comfort varies from 60.28 to 91.77 as shown in Fig. 9. The optimal palm direction is at $49^{\circ}$. In the can grasping situation, the palm direction can vary from $20^{\circ}$ to $80^{\circ}$ and the corresponding muscle comfort varies from 55.25 to 85.76 (Fig. 10). The optimal palm direction in this situation is at $61^{\circ}$.

The redundancy is then solved in these two cases by choosing the optimal solution. Moreover, as shown in these two figures, the choices resulted from this method appears to be the most natural one (coincides with human choice).

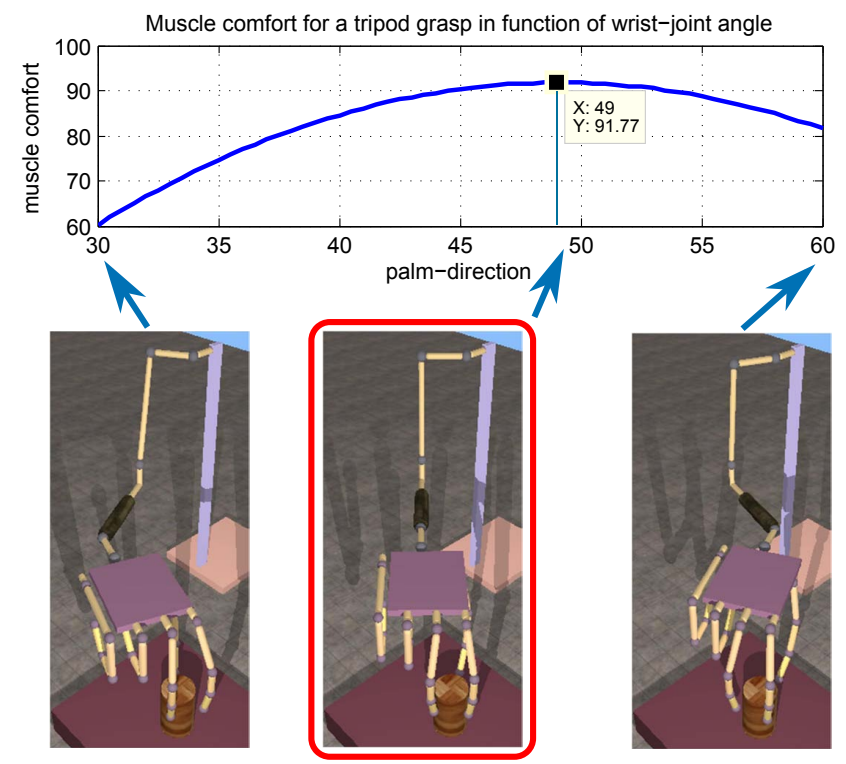

Fig. 9. Optimal choice of palm position in tripod grasp.

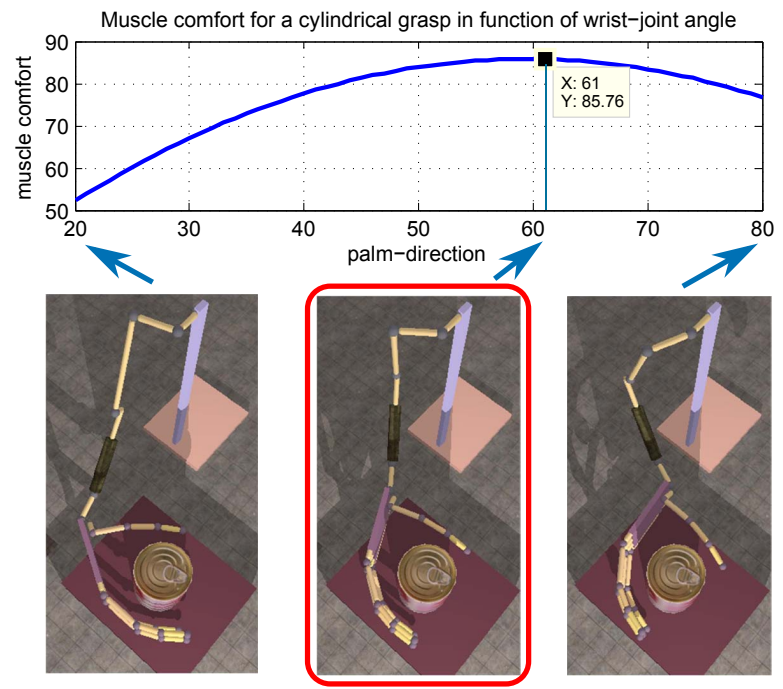

Fig. 10. Optimal choice of palm position in cylindrical grasp.

These two examples of grasping prove that the human natural gestures can be reproduced with an anthropomorphic hand by using the muscle comfort criterion.

\section{CONCLUSIONS AND FUTURE WORKS}

\section{A. Conclusion}

The tenodesis effect is a very interesting phenomenon that has an important impact on the choice of joint angle configuration in the grasping and in-hand manipulation tasks. By modeling the tendon constraints, reformulating them in the form of "muscle comfort" criterion and integrating this into a redundancy resolution framework, we succeeded in quantitatively deciphering the impact of this phenomenon on human grasping and in-hand manipulation. Moreover, this effect helped us solve the redundancy problem in different situations of grasp synthesis.

\section{B. Future Works}

The results obtained above prove that encoding the mechanical impacts on human grasping is very promising. The next step of the studies, apart from implementing the redundancy scheme to the real Shadow arm-hand robot, is to analyze the impact of the active muscle forces on the "natural characteristics" of the human gestures in grasping and in-hand manipulation. This study could potentially lead to the complete resolution of redundancy of anthropomorphic hands in grasping and in-hand manipulation with natural gestures as solutions.

\section{ACKNOWLEDGMENTS}

The authors gratefully acknowledge the contribution of Frank Quaine, GIPSA lab., Grenoble, France for the inspiring lecture on the biomechanical aspects of the human hand. The authors also acknowledge the team partner of the HANDLE project at FCTUC, Coimbra, Portugal, for their support during the recording process.

\section{REFERENCES}

[1] I. A. Kapandji, The Physiology of the Joints: Upper Limb: Volume 1. Churchill Livingstone, 1982.

[2] F.-C. Su, Y. Chou, C. Yang, G. Lin, and K. An, "Movement of finger joints induced by synergistic wrist motion," Clinical Biomechanics, 2005.

[3] A. V. Hill, "The heat of shortening and the dynamic constants of muscle," Proceedings of the Royal Society of London. Series B, Biological Sciences, 1938

[4] F. E. Zajac, "Muscle and tendon: properties, models, scaling, and application to biomechanics and motor control," Critical reviews in biomedical engineering, 1989

[5] A. Hollister, W. L. Buford, L. M. Myers, D. J. Giurintano, and A. Novick, "The axes of rotation of the thumb carpometacarpal joint." J. Orthop. Res., 1992.

[6] E. Y. S. Chao, K. N. An, W. P. Cooney, and R. L. Linscheid, Biomechanics of the Hand: A Basic Research Study. World Scientific Pub Co Inc, 1989.

[7] W. Weijs and B. Hillen, "Cross-sectional areas and estimated intrinsic strength of the human jaw muscles," Acta Morphol Neerl Scand, 1985.

[8] B. A. Garner and M. G. Pandy, "Estimation of musculotendon properties in the human upper limb," Annals of Biomedical Engineering, 2003.

[9] www.anykode.com.

[10] www.shadowrobot.com.

[11] H. Seraji and R. Colbaugh, "Singularity-robustness and taskprioritization in configuration control of redundant robots," in Proceedings of IEEE Conference on Decision and Control, 1990. 